\title{
THE HYDROXYL GROUPS INTERNAL ROTATIONS IN A METHANEDIOL MOLECULE
}

G.A.Pitsevich $^{\mathrm{a}^{*}}$, A.Ye.Malevich ${ }^{\mathrm{a}}$, V.V.Sapeshko ${ }^{\mathrm{b}}$

a Belarusian State University,

${ }^{\mathrm{b}}$ University of Illinois, Chicago, USA

*Corresponding author. E-mail address: pitsevich@bsu.by (George Pitsevich).

KEY WORDS: internal rotation, potential barriers, methanediol, potential energy surface, CBS, DVR, torsional spectra, molecular symmetry groups, methylene glycol, corformers.

Internal rotation of two of hydroxyl groups in a methanediol molecule is analyzed by constructing the $2 D$ surfaces of the potential energy, kinematic coefficients and dipole moment. The molecular characteristics in the nodes of $2 D$ grids with different densities for distinct pairs of dihedral angles of $\mathrm{O}-\mathrm{H}$ groups were calculated at the MP2/cc-pVQZ level of theory with optimization of the geometry for all other structural parameters. For optimized at the MP2/ccpVQZ level of theory geometries, the potential energy was additionally calculated at the $M P 2 / c c-p V T Z$ level of theory in the same grid nodes and then the extrapolation of energies in every node to the complete basis set limit was performed. The kinematic parameters were calculated using the formalism of the Wilson vectors. The Hamiltonian matrix was compiled using the DVR method. As a result of its diagonalization, the wave functions and energies of stationary torsional states were determined. The calculated torsional wave functions were classified by irreducible representations of the $C_{2 V}(M)$ molecular symmetry group. The frequencies and intensities of the torsional spectrum of a methanediol molecule were calculated at different temperatures for the trans- and cis-conformers. The tunneling frequencies for both conformers were calculated too.

\section{INTRODUCTION}

A methanediol (MD) molecule is the simplest representative of diols and is used in cosmetic and food industry. It has been considered for a long time that the only equilibrium configuration [1-3] is realized in the MD molecule. However, Rodler [4] found out for the first time that the molecule is featured by two equilibrium configurations. The trans-configuration, in which the hydroxyl groups are oppositely directed, is much more preferable energetically than the cis-configuration, in which the $\mathrm{O}-\mathrm{H}$ groups are almost parallel. A new wave of interest to the molecule was initiated recently by ascertaining the fact that MD plays an important role in the formation of atmospheric aerosols [5] as well as can be present in the interstellar space [6-8] and in the comets $[9,10]$. The search of this molecule in the cosmic space is complicated due to the absence of registered microwave and IR spectra of MD in the gas phase at present that is caused by low stability of the molecule. Indeed, the literature presents the experimental investigations of 
IR spectra of MD in the matrix isolation [11] as well as IR and Raman spectra in water solutions $[10,12-16]$, however the effect of the environment and possibility of formation of various associates reduces the significance of the results of these investigations for the identification of the MD molecules in the atmosphere and space. Therefore, the theoretical investigations of the spectral characteristics of this molecule are called for. MD stability in the gaseous phase was analysed for the first time within the up-to-date quantum-chemical methods in paper [7]. It was demonstrated that the molecule must be stable at temperatures below $100 \mathrm{~K}$. The effect of ammonia and formic acid on MD formation process in the reaction of formaldehyde with water is investigated in papers [10] and [5], respectively. The structural and spectral characteristics of MD and its associates in the water medium are analysed theoretically in paper [17]. The effect of the solvent polarity on the ratio of MD conformers is evaluated in paper [18]. In paper [19] the barriers of internal rotation of hydroxyl groups in MD were determined for the first time, the rotational constants were calculated and the pure rotational spectrum of the molecule was simulated. The IR spectrum of two conformers of the MD molecule in the harmonic approximation and for the first time in the anharmonic one was computed and presented in paper [8]. The last two papers are of the highest interest from the standpoint of use of their results for searching MD in the atmosphere and interstellar space. However, it is obvious that the success of such search is far from being ensured without analysis of internal rotation of hydroxyl groups in the molecule. As far as we know, results of such calculations are absent in literature up to this date.

In this paper, the energies of the stationary torsional states of the MD molecule were calculated at the complete basis set (CBS) limit using the MP2/cc-pVQZ level of theory. The torsional IR spectra of MD at various temperature were computed. The values of the tunnelling frequencies for two conformers of the molecule were estimated.

\section{CALCULATION DETAILS}

The equilibrium configurations of MD are presented in Fig.1 at the global (transconformer) and local (cis-conformer) energy minima as well as in the configuration being the initial one for performing the calculation of the 2D potential energy surface (PES). The first two configurations belong to the $C_{2}$ and $C_{S}$ symmetry point groups. The last one belongs to the $C_{2 V}$ symmetry point group and represents the transition state having one imaginary frequency in the calculated IR spectrum. The hydroxyl groups rotate around the $\mathrm{C}-\mathrm{O}$ bonds. Further we will be denoting the equivalent torsional coordinates as $\gamma$ and $\varphi$. The zero values of $\gamma$ and $\varphi$ were

chosen in the configuration presented on the right of Fig.1 where the hydroxyl atoms of hydrogen $\left(\mathrm{H}_{1}\right.$ and $\left.\mathrm{H}_{2}\right)$ lie in the plane, which is formed by the $\mathrm{O}_{5}-\mathrm{C}_{7}-\mathrm{O}_{6}$ atoms. 

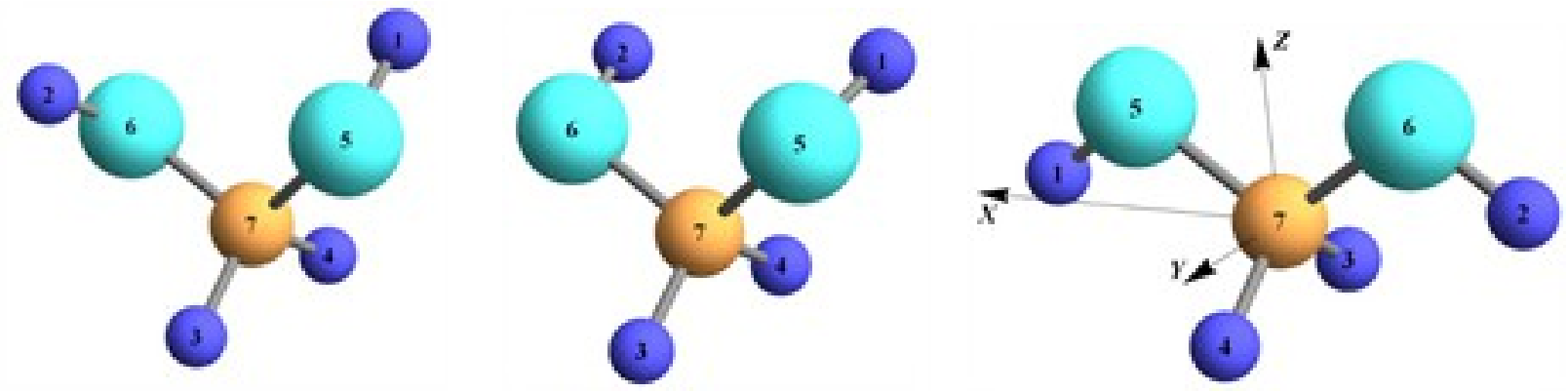

Figure 1. The equilibrium configuration of the trans- (on the left) and cis- (in the middle) conformers of MD as well as configuration being the initial one for performing the calculations of the $2 D$ PES (on the right).

The potential energy satisfies the condition $U(\gamma, \varphi)=U(-\gamma,-\varphi)$. Taking this relationship into account, the value of one of the coordinates should be varied within the range $\left(0-360^{\circ}\right)$ while the value of the second coordinate may be varied within the range $\left(0-180^{\circ}\right)$. Since the values of the energy of the torsional states were calculated using the DVR method, for proper symmetry account relative to the $\mathrm{O}_{5}-\mathrm{C}_{7}-\mathrm{O}_{6}$ plane, the values of the torsional coordinates on the $15^{*} 15$ grid were varied from $12^{\circ}$ to $348^{\circ}$ with the step of $24^{\circ}$ for $\gamma$ and from $12^{\circ}$ to $180^{\circ}$ with the step of $24^{\circ}$ for $\varphi$. When performing the calculations on the $45^{*} 45$ grid, the values of the torsional coordinates varied from $4^{\circ}$ to $356^{\circ}$ with the step of $8^{\circ}$ for $\gamma$ and from $4^{\circ}$ to $180^{\circ}$ with the step of $8^{\circ}$ for $\varphi$. In the nodes of the $15^{*} 15$ and $45 * 45$ equidistant grids, keeping fixed values of the torsional coordinates, MD geometry was optimized on all other coordinates using MP2 level of theory [20,21] and cc-pVQZ [22] basis set in the frame of the quantum-chemical package [23]. Configurations optimized in each node were used to calculate the kinematic coefficients using Wilson's vectors ( $\vec{s}$ - vectors) [24]. The Schrödinger equation with reduced dimensionality for the torsional vibrations of two hydroxyl groups can be written as follows [25-27]:

$$
\left[-F_{\gamma \gamma} \frac{\partial^{2}}{\partial \gamma^{2}}-F_{\varphi \varphi} \frac{\partial^{2}}{\partial \varphi^{2}}-F_{\gamma \varphi} \frac{\partial^{2}}{\partial \gamma \partial \varphi}+U(\gamma, \varphi)\right] \Psi(\gamma, \varphi)=E \Psi(\gamma, \varphi)
$$

The kinematic parameters can be determined from the following relations [25-27]:

$$
\begin{gathered}
F_{\gamma \gamma}=B_{H}\left[\left(\vec{s}_{1}^{\gamma}\right)^{2}+\left(\vec{s}_{3}^{\gamma}\right)^{2}+\left(\vec{s}_{4}^{\gamma}\right)^{2}\right]+B_{O}\left[\left(\vec{s}_{5}^{\gamma}\right)^{2}+\left(\vec{s}_{6}^{\gamma}\right)^{2}\right]+B_{C}\left(\vec{s}_{7}^{\gamma}\right)^{2} \\
F_{\varphi \varphi}=B_{H}\left[\left(\vec{s}_{2}^{\varphi}\right)^{2}+\left(\vec{s}_{3}^{\varphi}\right)^{2}+\left(\vec{s}_{4}^{\varphi}\right)^{2}\right]+B_{O}\left[\left(\vec{s}_{5}^{\varphi}\right)^{2}+\left(\vec{s}_{6}^{\varphi}\right)^{2}\right]+B_{C}\left(\vec{s}_{7}^{\varphi}\right)^{2} \\
F_{\gamma \varphi}=B_{H}\left[2\left(\vec{s}_{3}^{\gamma} \cdot \vec{s}_{3}^{\varphi}\right)+2\left(\vec{s}_{4}^{\gamma} \cdot \vec{s}_{4}^{\varphi}\right)\right]+B_{O}\left[2\left(\vec{s}_{5}^{\gamma} \cdot \vec{s}_{5}^{\varphi}\right)+2\left(\vec{s}_{6}^{\gamma} \cdot \vec{s}_{6}^{\varphi}\right)\right]+B_{C} 2\left(\vec{s}_{7}^{\gamma} \cdot \vec{s}_{7}^{\varphi}\right)
\end{gathered}
$$

Here $\vec{s}_{N}^{x}$ is Wilson's vector for the coordinate $x=\{\gamma, \varphi\}$ and for the atom with the number $N$ (the numbering of atoms corresponds to Fig.1 (on the right)), $B_{X}=\frac{\hbar^{2}}{2 M_{X} l_{0}^{2}} ; X \in\{H, C, O\} \hbar$ is 
the Plank's constant, $l_{0}=1 \AA, M_{H}, M_{C}, M_{O}$ are the corresponding masses of hydrogen, carbon and oxygen atoms.

For the MD molecule geometries that were optimized at all the grid nodes at the MP2/ccpVQZ level of theory, potential energy values were computed at the MP2/cc-pVTZ [28] level of theory without optimization. Then energy values of every node were extrapolated over the two datasets (MP2/cc-VTZ and MP2/cc-pVQZ) to the CBS limit using known formulas [29,30].

Since the Cartesian coordinate system changed its orientation in relation to the molecule as the hydroxyl groups rotates, components of the dipole moment vector at each node of the 2D grid were recalculated for the Cartesian coordinate system related to the molecular skeleton, in which the $\mathrm{Z}$ axis was directed along $\mathrm{O}_{5}-\mathrm{C}_{7}-\mathrm{O}_{6}$ angle bisector, the $\mathrm{X}$ axis lied in the plane of the $\mathrm{O}_{5}-\mathrm{C}_{7}-\mathrm{O}_{6}$ atoms and the $\mathrm{Y}$ axis supplemented the $\mathrm{X}$ and $\mathrm{Y}$ to the right-hand triple and was, therefore, normal to the plane of three heavy atoms.

To solve the Schrödinger equation (1) the DVR method was used [31-36]. Hamiltonian matrix was calculated by the formula:

$$
H_{(i, j)\left(i^{\prime}, j^{\prime}\right)}=-F_{\gamma \gamma}\left(\gamma_{i}, \varphi_{j}\right) D_{i i^{\prime}}^{\gamma \gamma} \delta_{j j^{\prime}}-F_{\varphi \varphi}\left(\gamma_{i}, \varphi_{j}\right) \delta_{i i^{\prime}} D_{j j^{\prime}}^{\varphi \varphi}-F_{\gamma \varphi}\left(\gamma_{i}, \varphi_{j}\right) D_{i i^{\prime}}^{\gamma} D_{j j^{\prime}}^{\varphi}+U\left(\gamma_{i}, \varphi_{j}\right) \delta_{i i^{\prime}} \delta_{j j^{\prime}}
$$

Here:

$$
\begin{aligned}
& D^{x y}=\tilde{D}^{x} D^{y} ; \quad D_{i i^{\prime}}^{x}=\frac{(-1)^{i^{\prime}-i}}{2 \sin \left[\frac{\pi\left(i^{\prime}-i\right)}{N_{X}}\right]} ; D_{i i}^{x}=0 ; \\
& x \in(\gamma, \varphi) ; \quad i^{\prime}, i \in\left\{1 \div N_{X}\right\} ; N_{X}=15 \text { or } 45 ;
\end{aligned}
$$

Squares of matrix elements of the dipole moments operator were found using [37] and the following formula:

$$
p_{i f}^{2}=\sum_{k=x, y, z}\left(\int_{\gamma=0}^{2 \pi} \int_{\varphi=0}^{2 \pi} \Psi_{i}(\gamma, \varphi) p_{k}(\gamma, \varphi) \Psi_{f}(\gamma, \varphi) d \gamma d \varphi\right)^{2}
$$

Here $p_{x}, p_{y}, p_{z}$ are the dipole moment vector components in the molecular fixed Cartesian coordinate system, $\Psi_{i}(\gamma, \varphi), \Psi_{f}(\gamma, \varphi)$ are the torsional wave functions in the initial $(i)$ and final $(f)$ states.

\section{SYMMETRY ANALISYS}

As was mention above, the MD molecule is a non-rigid object having two equilibrium configurations belonging to the $\mathrm{C}_{2}$ and $\mathrm{C}_{\mathrm{s}}$ symmetry point groups. Both conformers can tunnel to the equivalent configurations passing through the transition state. It is natural to classify stationary torsional states using the appropriate molecular symmetry group [38-40]. This group includes only feasible permutation and permutation-inversion symmetry elements. One can see 
that these elements are E, $(12)(34)(56),(34)^{*}$ and $(12)(56)^{*}$. These four elements form the $\mathrm{C}_{2 \mathrm{~V}}(\mathrm{M})$ molecular symmetry group, which is isomorph to the $\mathrm{C}_{2 \mathrm{~V}}$ point group symmetry [40]. It is important that all four symmetry elements are inherent to both equilibrium conformers $\left(\mathrm{C}_{2}\right.$ and $\mathrm{C}_{\mathrm{S}}$ ) of the MD molecule. In addition, one can see that MD molecule transition state has the $\mathrm{C}_{2 \mathrm{~V}}$ symmetry, which is consistent with the fact that the $\mathrm{C}_{2 \mathrm{~V}}(\mathrm{M})$ molecular symmetry group is isomorph to the $\mathrm{C}_{2 \mathrm{~V}}$ point group. The irreducible representations of the $\mathrm{C}_{2 \mathrm{~V}}(\mathrm{M})$ molecular symmetry group are shown in Table 1 .

Table 1 The table of irreducible representations of the $\mathrm{C}_{2 \mathrm{~V}}(\mathrm{M})$ molecular symmetry group [40]

\begin{tabular}{|c|c|c|c|c|}
\hline $\mathrm{G}_{4}$ & $\mathrm{E}$ & $(12)(34)(56)$ & $(34)^{*}$ & $(12)(56)^{*}$ \\
\hline Short name & $\mathrm{P}_{1}$ & $\mathrm{P}_{2}$ & $\mathrm{P}_{3}$ & $\mathrm{P}_{4}$ \\
\hline $\mathrm{A}_{1}$ & 1 & 1 & 1 & 1 \\
\hline $\mathrm{A}_{2}$ & 1 & 1 & -1 & -1 \\
\hline $\mathrm{B}_{1}$ & 1 & -1 & 1 & -1 \\
\hline $\mathrm{B}_{2}$ & 1 & -1 & -1 & 1 \\
\hline
\end{tabular}

It is important to know that the symmetry elements of the $2 \mathrm{D}$ surfaces of the potential energy, wave functions, components of the dipole momentum and kinematic coefficients are connected with symmetry elements of the $\mathrm{C}_{2 \mathrm{~V}}(\mathrm{M})$ group. Let's call the plane which is perpendicular to the coordinate plane $\gamma, \varphi$ and intersect it along diagonal $\left(0^{\circ}, 0^{0}-360^{\circ}, 360^{\circ}\right)$ plane 1. The plane which is perpendicular to the coordinate plane $\gamma, \varphi$ and intersects it along diagonal $\left(0^{0}, 360^{\circ}-360^{\circ}, 0^{\circ}\right)$ will be plane 2 . The intersection of these two planes will be the $\mathrm{C}_{2}$ symmetry axis. Then these elements will be connected with symmetry elements of the $\mathrm{C}_{2 \mathrm{~V}}(\mathrm{M})$ group according to data from Table 2. In addition, the transformation of the torsional coordinates $\gamma, \varphi$ under the action of the $\mathrm{C}_{2 \mathrm{~V}}(\mathrm{M})$ symmetry operations includes in Table 2 too.

Table 2. The transformation of the torsional coordinates under the action of the $\mathrm{C}_{2 \mathrm{~V}}(\mathrm{M})$ symmetry operations and interconnection of the symmetry elements of the $2 \mathrm{D}$ surfaces of the PES, wave functions with the $\mathrm{C}_{2 \mathrm{~V}}(\mathrm{M})$ symmetry operations.

\begin{tabular}{|c|c|c|c|c|}
\hline $\mathrm{C}_{2 \mathrm{~V}}(\mathrm{M})$ & $\mathrm{E}$ & $(12)(34)(56)$ & $(34)^{*}$ & $(12)(56)^{*}$ \\
\hline Short name & $\mathrm{P}_{1}$ & $\mathrm{P}_{2}$ & $\mathrm{P}_{3}$ & $\mathrm{P}_{4}$ \\
\hline $\begin{array}{c}\text { Transformations of } \\
\text { the torsional } \\
\text { coordinates }\end{array}$ & $\gamma, \varphi$ & $\varphi, \gamma$ & $-\gamma,-\varphi$ & $-\varphi,-\gamma$ \\
\hline $\begin{array}{c}\text { Symmetry elements } \\
\text { of the 2D surfaces }\end{array}$ & $\mathrm{E}$ & Reflection in plane 1 & $\begin{array}{c}\text { Rotation around } \mathrm{C}_{2} \\
\text { axis }\end{array}$ & Reflection in plane 2 \\
\hline
\end{tabular}

According to the data from Table 2 one can get, for example: $P_{3}(\gamma, \varphi)=(-\gamma,-\varphi)$. 
Now it is obvious that using symmetry elements of the $\mathrm{C}_{2 \mathrm{~V}}(\mathrm{M})$ molecular symmetry group (see Table 1 [40]) and data from Table 2 it is possible to classify any torsional wave function $\Psi_{k}(\gamma, \varphi)$ as well as any other molecular property like, for example, PES by visual analysis or with help of the next formulas:

$$
\begin{aligned}
& \text { if }\left|\Psi_{k}(\gamma, \varphi)-P_{j} \Psi_{k}(\gamma, \varphi)\right|=\left|\Psi_{k}(\gamma, \varphi)-\Psi_{k}\left(P_{j}(\gamma, \varphi)\right)\right|=0 \Rightarrow \chi_{i, j}=1 \\
& \text { if }\left|\Psi_{k}(\gamma, \varphi)+P_{j} \Psi_{k}(\gamma, \varphi)\right|=\left|\Psi_{k}(\gamma, \varphi)+\Psi_{k}\left(P_{j}(\gamma, \varphi)\right)\right|=0 \Rightarrow \chi_{i, j}=-1
\end{aligned}
$$

Gathering four characters of the irreducible representation $\Gamma_{i}\left(\chi_{i, l} \equiv 1, \chi_{i, 2}, \chi_{i, 3}, \chi_{i, 4}\right)$ it is possible to determine one of four $\left(\mathrm{A}_{1}, \mathrm{~A}_{2}, \mathrm{~B}_{1}, \mathrm{~B}_{2}\right)$ symmetry species of the $k$ - torsional state.

\section{DISCUSSION OF THE RESULTS}

The 2D PES of the MD molecule calculated at the MP2/cc-pVQZ level of theory is presented in Fig.2.

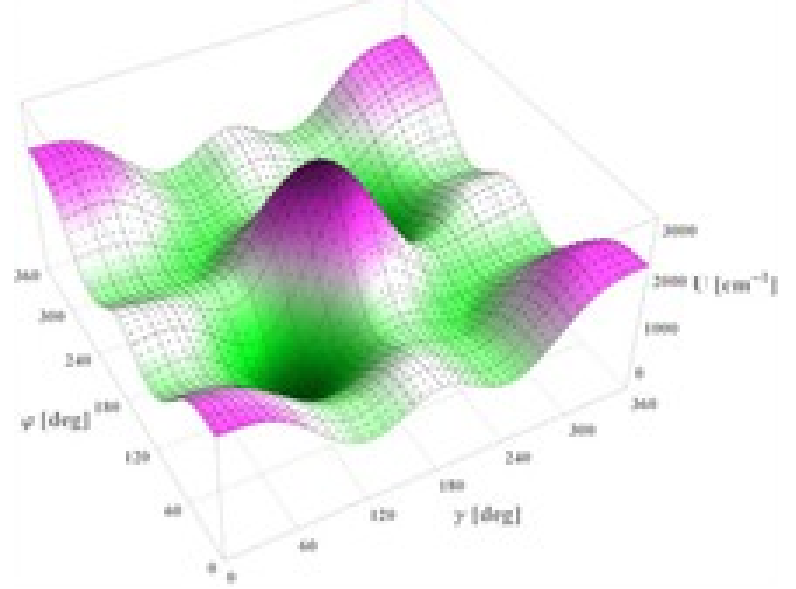

Fig.2. 2D PES of the MD molecule calculated at the MP2/cc-pVQZ level of theory. The 2D uniform grid nodes at which the potential energy was computed are shown by points in the $2 D$ PES.

The two global energy minima and the two local ones corresponding to the trans- and cisconformers of MD in two structurally equivalent configurations are well visible in Fig.2. Fig.3 presents the 2D surfaces of the kinematic coefficients. It should be noted that the 2D surfaces of the kinematic coefficients $F_{\gamma \gamma}$ and $F_{\varphi \varphi}$ are equivalent. 

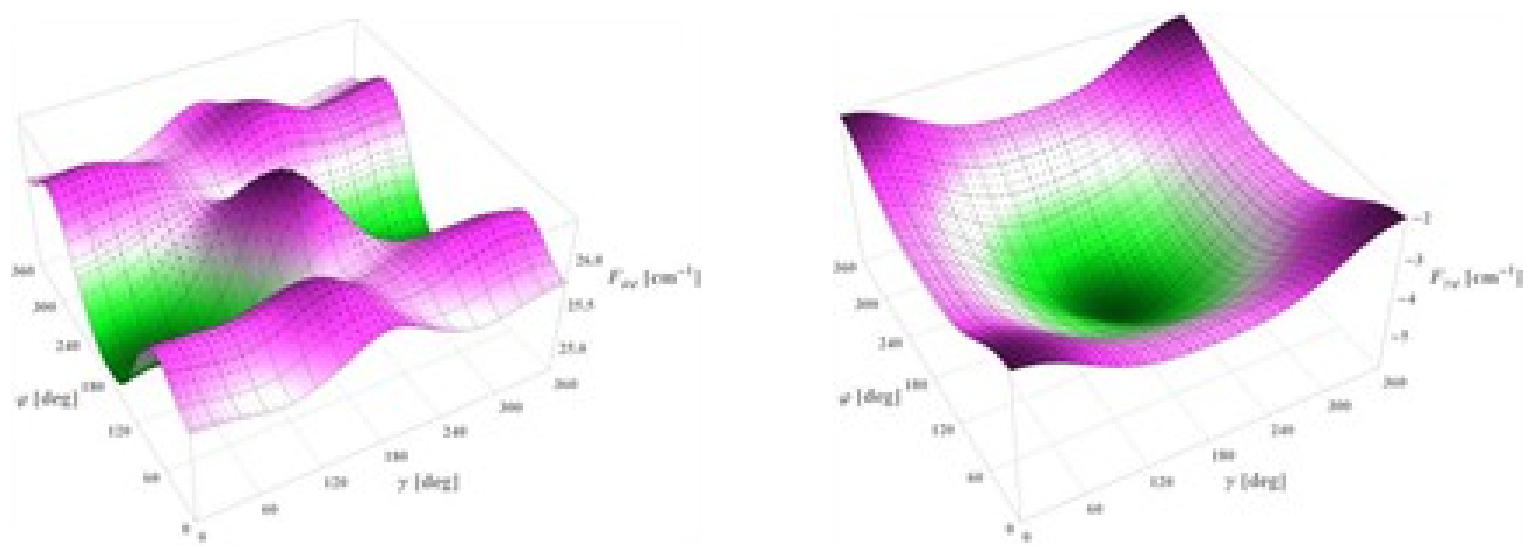

Figure 3. 2D surfaces of the kinematic coefficients $F_{\varphi \varphi}$ and $F_{\gamma \gamma}$ (on the left), and $F_{\gamma \varphi}$ (on the right) calculated at the MP2/cc-pVQZ level of theory.

Fig.4 presents the 2D surfaces of the three components of the dipole moment of the MD molecule calculated at the MP2/cc-pVQZ level of theory.


Figure 4. The 2D surfaces of the three components of the dipole moment of the MD molecule calculated at the MP2/cc-pVQZ level of theory (x-component on the left, y-component in the middle and z-component on the right). The values of the components are calculated in relation to the molecularly fixed Cartesian coordinate system, the location of which relatively to the molecule is described in section 2.

The 2D PES of the MD molecule as well as the 2D surfaces of all three kinematic coefficients are symmetrical with respect to all the symmetry elements of the $\mathrm{C}_{2 \mathrm{~V}}(\mathrm{M})$ group and, therefore, are transformed according to the fully symmetric representation of $A_{1}$. The components of the dipole moment $p_{X}, p_{Y}$ and $p_{Z}$ are transformed by the irreducible representations $B_{1}, B_{2}$, and $A_{1}$ of the $C_{2} v(M)$ molecular symmetry group. Using the data given in Table 2 and formulas (8) and (9), one can also classify torsional wave functions by the irreducible representations of the $\mathrm{C}_{2 \mathrm{~V}}(\mathrm{M})$ groups.

The values of the energies of the stationary torsional states of the MD molecule calculated at the MP2/cc-pVTZ and MP2/cc-pVQZ level of theory and in the CBS limit on the 2D $45^{*} 45$ and $15 * 15$ grids (in the last case) are presented in Table 3 (columns $3-5$ ). In columns 6-9 of Table 3, torsional frequencies of the transitions from the ground level are presented. We 
were also able to determine the torsional quantum numbers for several lowest torsional states. The $n_{a s}$ and $n_{s}$ in 10 and 11 columns of Table 3 are the quantum numbers which specify antisymmetric and symmetric torsional vibrations in trans- and cis-conformers (see the second column in Table 3). The symmetry species of the torsional wave functions are represented in the last column of Table 3 .

Table 3. Values of the energies in $\left[\mathrm{cm}^{-1}\right]$ and symmetry of the stationary torsional states of the MD molecule calculated at the MP2/cc-pVTZ and MP2/cc-pVQZ level of theory and in the CBS limit.

\begin{tabular}{|c|c|c|c|c|c|c|c|c|c|c|c|}
\hline $\begin{array}{l}\text { Level's } \\
\text { number }\end{array}$ & Configuration & $\begin{array}{c}\text { MP2/cc- } \\
\text { pVTZ } \\
45 * 45\end{array}$ & $\begin{array}{c}\text { MP2/cc- } \\
\text { pVQZ } \\
45^{*} 45\end{array}$ & $\begin{array}{c}\text { CBS } \\
45 * 45\end{array}$ & $\begin{array}{c}\text { MP2/cc- } \\
\text { pVTZ } \\
45^{*} 45\end{array}$ & $\begin{array}{c}\text { MP2/cc- } \\
\text { pVQZ } \\
45^{* 45}\end{array}$ & $\begin{array}{c}\text { CBS } \\
45^{*} 45\end{array}$ & $\begin{array}{c}\text { CBS } \\
15 * 15\end{array}$ & $n_{a s}$ & $n_{s}$ & $\begin{array}{c}\text { Symmetry } \\
\text { species }\end{array}$ \\
\hline 1 & trans & 282.5 & 277.9 & 273.9 & 0 & 0 & 0 & 0 & 0 & 0 & $\mathrm{~A}_{1}$ \\
\hline 2 & trans & 282.5 & 277.9 & 273.9 & $1.87 * 10^{-6}$ & $1.99 * 10^{-6}$ & $2.11 * 10^{-6}$ & $1.57 * 10^{-4}$ & 0 & 0 & $\mathrm{~A}_{2}$ \\
\hline 3 & trans & 639.8 & 625.2 & 613.8 & 357.3 & 347.3 & 339.9 & 328.1 & 1 & 0 & $\mathrm{~B}_{2}$ \\
\hline 4 & trans & 639.9 & 625.2 & 613.8 & 357.3 & 347.3 & 339.9 & 328.1 & 1 & 0 & $\mathrm{~B}_{1}$ \\
\hline 5 & trans & 693.9 & 681.5 & 671.7 & 411.4 & 403.6 & 397.8 & 390.7 & 0 & 1 & $\mathrm{~A}_{1}$ \\
\hline 6 & $\operatorname{trans}$ & 693.9 & 681.5 & 671.7 & 411.4 & 403.6 & 397.8 & 390.7 & 0 & 1 & $\mathrm{~A}_{2}$ \\
\hline 7 & $\operatorname{trans}$ & 960.2 & 935.8 & 917.1 & 677.7 & 657.9 & 643.2 & 655.4 & 2 & 0 & $\mathrm{~A}_{1}$ \\
\hline 8 & trans & 960.2 & 935.8 & 917.1 & 677.7 & 657.9 & 643.2 & 655.4 & 2 & 0 & $\mathrm{~A}_{2}$ \\
\hline 9 & trans & 1016.1 & 992.0 & 973.5 & 733.6 & 714.1 & 699.6 & 707.5 & 1 & 1 & $\mathrm{~B}_{2}$ \\
\hline 10 & trans & 1016.1 & 992.0 & 973.5 & 733.6 & 714.1 & 699.6 & 707.5 & 1 & 1 & $\mathrm{~B}_{1}$ \\
\hline 11 & trans & 1082.9 & 1062.2 & 1046.2 & 800.4 & 784.3 & 772.3 & 761.6 & 0 & 2 & $\mathrm{~A}_{1}$ \\
\hline 12 & trans & 1082.9 & 1062.2 & 1046.2 & 800.4 & 784.3 & 772.3 & 761.7 & 0 & 2 & $\mathrm{~A}_{2}$ \\
\hline 13 & cis & 1113.8 & 1084.7 & 1062.5 & 831.2 & 806.7 & 788.6 & 786.8 & 0 & 0 & $\mathrm{~B}_{2}$ \\
\hline 14 & cis & 1113.8 & 1084.9 & 1062.8 & 831.4 & 807.0 & 788.9 & 787.1 & 0 & 0 & $\mathrm{~A}_{1}$ \\
\hline 15 & cis + trans & 1220.2 & 1183.3 & 1155.1 & 937.6 & 905.3 & 881.2 & 862.8 & $1+1$ & 0 & $\mathrm{~B}_{1}$ \\
\hline 16 & cis & 1231.7 & 1197.3 & 1169.9 & 949.2 & 919.3 & 896.0 & 870.8 & 0 & 1 & $\mathrm{~A}_{2}$ \\
\hline 17 & $\operatorname{trans}$ & 1239.0 & 1199.5 & 1171.1 & 956.5 & 921.6 & 897.2 & 889.8 & 3 & 0 & $\mathrm{~B}_{2}$ \\
\hline 18 & cis + trans & 1258.2 & 1221.3 & 1193.8 & 975.7 & 943.3 & 919.9 & 910.2 & $1+1$ & 0 & $\mathrm{~B}_{1}$ \\
\hline 19 & trans & 1283.5 & 1247.2 & 1219.3 & 1001.0 & 969.3 & 945.4 & 912.2 & 2 & 1 & $\mathrm{~A}_{1}$ \\
\hline 20 & trans & 1290.7 & 1254.5 & 1227.0 & 1008.1 & 976.6 & 953.1 & 925.3 & 2 & 1 & $\mathrm{~A}_{2}$ \\
\hline 21 & cis & 1337.9 & 1292.7 & 1259.2 & 1055.4 & 1014.8 & 985.3 & 985.5 & 2 & 0 & $\mathrm{~A}_{1}$ \\
\hline 22 & cis & 1340.6 & 1295.5 & 1261.9 & 1058.1 & 1017.6 & 988.0 & 988.3 & 2 & 0 & $\mathrm{~A}_{2}$ \\
\hline 23 & trans & 1361.1 & 1323.9 & 1293.4 & 1078.6 & 1046.0 & 1019.5 & 1026.6 & 1 & 2 & $\mathrm{~B}_{1}$ \\
\hline 24 & $\operatorname{trans}$ & 1364.2 & 1329.5 & 1303 & 1081.7 & 1051.6 & 1029.1 & 1031.2 & 1 & 2 & $\mathrm{~B}_{2}$ \\
\hline 25 & cis + trans & 1396.9 & 1347.7 & 1311 & 1114.4 & 1069.8 & 1037 & 1038.7 & - & - & $\mathrm{A}_{2}$ \\
\hline 26 & cis + trans & 1399.7 & 1352.9 & 1320.3 & 1117.1 & 1075.0 & 1046.4 & 1039.7 & - & - & $\mathrm{B}_{1}$ \\
\hline
\end{tabular}

As seen from the data in Table 3, calculated in MP2/cc-pVTZ and MP2/cc-pVQZ approximations values of the energies and frequencies of the torsional transitions from the ground state converge to the respective values obtained in the CBS limit. It can be also noted that the values of frequencies of the torsional transitions from the ground state obtained in the CBS limit for the $15^{*} 15$ and $45^{*} 45$ grids overall agree well with each other. However, the results obtained using the denser grid have to be more exact in comparison to results for the less dense grid. It is also interesting to compare the values of the frequencies of the lowest-frequency torsional transitions obtained in the harmonic and anharmonic approximations with the data obtained by the solution of the Schrödinger equation. To do this IR spectra of the two MD conformers were calculated in harmonic and anharmonic approximations using MP2/cc-pVQZ level of theory. For the trans-conformer, the values of the frequencies of the torsional vibrations in the harmonic and anharmonic approximations were 371 and $377 \mathrm{~cm}^{-1}$ (harmonic 
approximation), and 209 and $263 \mathrm{~cm}^{-1}$ (anharmonic approximation). The respective values of the frequencies obtained by solving the Schrödinger equation (MP2/cc-VQZ) were 347 and $404 \mathrm{~cm}^{-}$ ${ }^{1}$. For the cis-conformer, the values of the frequencies of the torsional vibrations in the harmonic and anharmonic approximations were 192 and $387 \mathrm{~cm}^{-1}$ (harmonic approximation), and -275 and $157 \mathrm{~cm}^{-1}$ (anharmonic approximation). The respective value of the low frequency vibration obtained by solving the Schrödinger equation (MP2/cc-VQZ) was $112,6 \mathrm{~cm}^{-1}$. The symmetric high frequency torsional vibration in cis-conformer is mixed with torsional vibrations of the trans-conformer and cannot be directly compared with the vibration calculated in harmonic and anharmonic approximations. As one can see, in the case of trans-conformer the values of frequencies of the torsional vibrations obtained in the harmonic approximation are closer to the grid calculations than the anharmonic ones. In the case of cis-conformer, the anharmonic approximation is failed. The inability of the anharmonic approximation to predict the vibrational frequencies in the case of very anharmonic PES was noted in a number of publications [41-44].

Now we will consider results of calculations of the tunnelling frequencies in the transand cis-conformers. Having excluded the results of the calculation in the MP2/cc-pVTZ approximation, it may be noted that the results obtained in the MP2/cc-pVQZ approximation and in the CBS limit agree well. For the trans-conformer, the values of these frequencies are $1.99 * 10^{-}$ ${ }^{6}$ and $2.11 * 10^{-6} \mathrm{~cm}^{-1}$ or 0.0597 and $0.0633 \mathrm{MHz}$, respectively. For the cis-conformer, the values of the tunnelling frequencies in the MP2/cc-pVQZ approximation and in the CBS limit are 0.234 and $0.265 \mathrm{~cm}^{-1}$ or 7015 and $7945 \mathrm{MHz}$, respectively. The values of the matrix elements of the dipole moment operator calculated in the MP2/cc-pVTZ and MP2/cc-pVQZ approximations and in the CBS limit are presented in the Additional materials.

To calculate the intensities of the torsional IR spectrum, it is also necessary to consider the symmetry of the torsional wave functions with respect to the permutation of the chemically equivalent atoms in the MD molecule. Since ${ }^{16} \mathrm{O}$ and ${ }^{12} \mathrm{C}$ atoms have zero spin they can be excluded from further consideration. First of all, one has to take into account symmetry properties of the total, torsional and spin wave functions with respect to symmetry elements of the $\mathrm{C}_{2 \mathrm{~V}}(\mathrm{M})$ molecular symmetry group which do not include the inversion operation $[40,45]$. These are E and (12)(34)(56) symmetry operations. Due to the last one interchanges even number of the fermion nuclei (protons) the total wave function must be symmetric with respect to these two symmetry operations and consequently has to belong to $A_{1}$ or $A_{2}$ symmetry species. The torsional wave functions that belong to the $A_{1}$ and $A_{2}$ symmetry species are symmetric with respect to interchange two pairs of fermion nuclei while the ones that belong to the $B_{1}$ and $B_{2}$ symmetry species are antisymmetric. The reducible representation of the hydrogen's nuclear spin 
functions can be decomposed into irreducible representations of the $\mathrm{C}_{2 \mathrm{~V}}(\mathrm{M})$ molecular symmetry group as follows [46]:

$$
\Gamma_{C_{2 V}(M)}^{\text {spin }}=9 A_{1}+1 A_{2}+3 B_{1}+3 B_{2}
$$

Thereby, the torsional wave functions must be combined with the spin wave functions as it is shown in Table 4.

Table 4 Possible combinations of the torsional and spin wave functions for the MD molecule.

\begin{tabular}{|c|c|c|}
\hline Symmetry species of the torsional wave functions & $\mathrm{A}_{1}, \mathrm{~A}_{2}$ & $\mathrm{~B}_{1}, \mathrm{~B}_{2}$ \\
\hline Symmetry species of the spin wave functions & $\mathrm{A}_{1}, \mathrm{~A}_{2}$ & $\mathrm{~B}_{1}, \mathrm{~B}_{2}$ \\
\hline Symmetry species of the total wave functions & $\mathrm{A}_{1}, \mathrm{~A}_{2}$ & $\mathrm{~A}_{1}, \mathrm{~A}_{2}$ \\
\hline The degeneration of the torsional wave functions (g) & $9+1$ & $3+3$ \\
\hline Specificator (s) & 1 & -1 \\
\hline
\end{tabular}

As one can see from Table 4 , the torsional wave functions that belong to $A_{1}$ and $A_{2}$ symmetry species are tenfold degenerate, while the torsional wave functions that belong to $B_{1}$ and $B_{2}$ symmetry species are sixfold degenerate. In addition, the symmetry of the spin wave functions must be saved during the torsional transitions. Relative intensities of the IR bands for torsional transitions from the initial state $i$ to the final state $f$ were calculated using the next formulas [40,47-49]:

$$
\begin{gathered}
I_{i \rightarrow f}=\frac{\operatorname{Const} \cdot \tilde{v}_{i f} \cdot \frac{\left|s_{i}+s_{f}\right|}{2} \cdot g_{i} \cdot\left[e^{-\frac{E_{i}-E_{1}}{k T}}-e^{-\frac{E_{f}-E_{1}}{k T}}\right]}{Q(T)} p_{i f}^{2} \\
Q(T)=\sum_{i} g_{i} e^{-\frac{E_{i}-E_{1}}{k T}} ; \quad \text { (12) }
\end{gathered}
$$

where $g_{i}$ - the degeneracy of the initial torsional state value of which is consistent with the type of symmetry of the torsional wave function in accordance with the data of Tables 3 and $4, s_{i}$ and $s_{f}$ - the initial and final state specifier value of which, as indicated in Table 4 , is 1 for the $\mathrm{A}_{1}$ и $\mathrm{A}_{2}$ torsional states, and is -1 for $\mathrm{B}_{1}$ и $\mathrm{B}_{2}$ torsional states, $Q(T)$ - partition function.

With the use of these data, the relative intensities of the IR absorption bands conditioned by the torsional transitions in the MD molecule at different temperatures were calculated from the formula (11). Fig. 5 presents the IR spectrum of the MD molecule at the temperatures of 300 and $30 \mathrm{~K}$ calculated in the CBS limit.

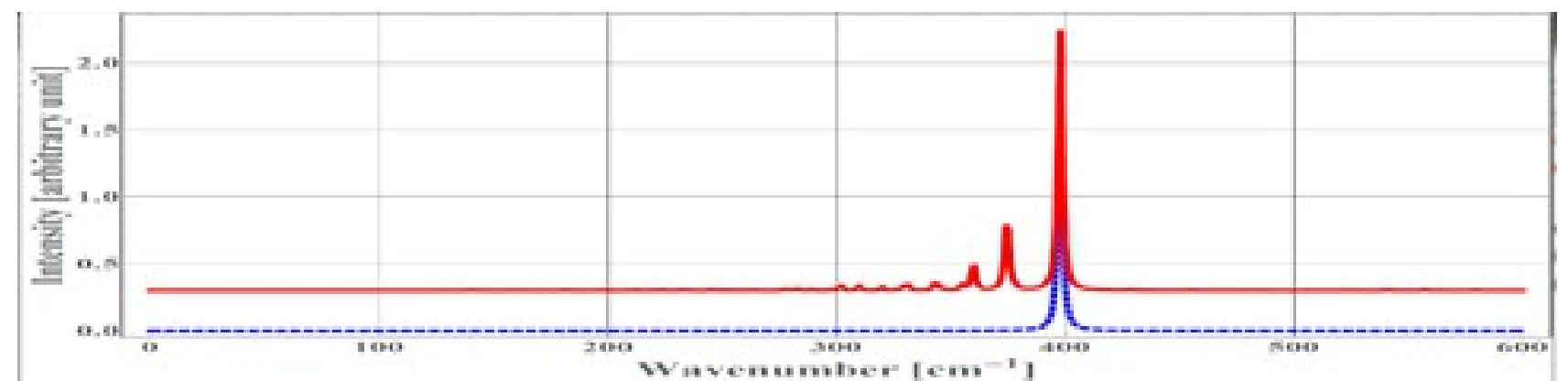


Figure 5. Torsional IR spectra of the MD molecule at the temperatures of $300 \mathrm{~K}$ (red colour) and $30 \mathrm{~K}$ (blue colour) calculated in the approximation of the CBS limit.

As seen from the upper spectrum in Fig.5, only three intensive IR bands expected for the MD molecule at room temperature. The most intense absorption band with a maximum of about $397.8 \mathrm{~cm}^{-1}$ is due to transitions from the first to the fifth and from the second to the sixth torsional energy levels. In both cases, this leads to the excitation of a symmetric torsional vibration in the trans- conformer of MD. Note that the optical transitions from the first two states to the third and fourth excited states, which would correspond to the excitation of the antisymmetric torsional state in the trans-conformer, are forbidden since the combining states belong to different multiplicities. Another two less intense absorption bands with frequencies of maxima near 374.5 and $359.8 \mathrm{~cm}^{-1}$ are due to transitions $5 \Rightarrow 11,6 \Rightarrow 12$ and $3 \Rightarrow 9,4 \Rightarrow 10$ respectively. Obviously, at low temperatures, torsional states 3-6 are not populated and, therefore, only one intensive absorption band of $397.8 \mathrm{~cm}^{-1}$ is expected in the calculated torsional spectrum of trans-conformer of MD at $30 \mathrm{~K}$.

If the potential barriers are sufficiently high, it becomes possible to make the cisconformer stable at temperatures below $30 \mathrm{~K}$. To estimate the height of the potential barrier in case of transition from the cis-conformer to the trans-conformer using Mathematica [37] package, the potential energy of MD was determined at every point for the values $\gamma$ and $\varphi$ specified within the range $0-2 \pi$ with the help of interpolation. The map of gradients on the potential energy surface is presented in Fig. 6.

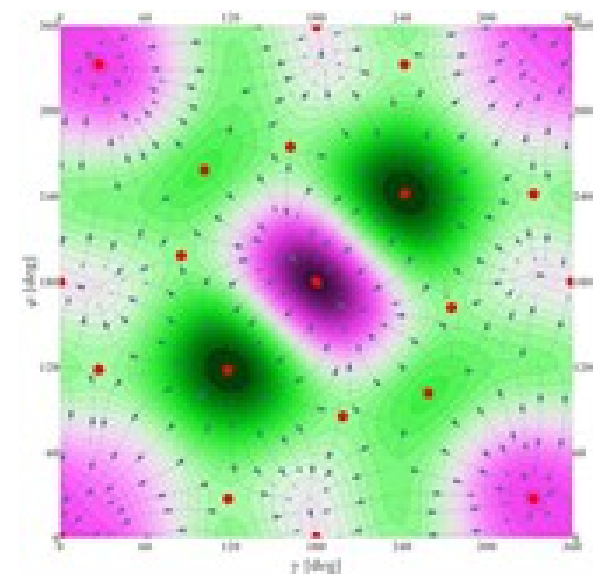

Figure 6. Map of the energy gradient directions. The decrease of energy contributes to the strengthening of the green colour while the increase of the energy contributes to the intensification of the pink colour. The global and local minima along with saddle points are marked by red dots.

Using Fig.6, one can find out that the minimum potential barrier, which must be overcomed by the molecule during the transition from the cis- to the trans-configuration is 327 $\mathrm{cm}^{-1}$. However, if one takes into account zero point energy for cis-conformer $\left(258 \mathrm{~cm}^{-1}\right)$ it 
becomes clear that the potential barrier is only about $70 \mathrm{~cm}^{-1}$. This fact makes the cis-conformer of the MD molecule potentially unstable. At $30 \mathrm{~K}$ more than $8 \%$ of the cis-conformers will have the kinetic energy equal or higher than $70 \mathrm{~cm}^{-1}$. On the other hand, at $10 \mathrm{~K}$ only $0,062 \%$ of the cis-conformers will have the kinetic energy equal or higher than $70 \mathrm{~cm}^{-1}$. Furthermore, to convert this kinetic energy to internal energy successful collision needed. However, in the low density interstellar space collisions don't happen often. Anyway, we have calculated the torsional spectrum of the cis-transformer at the temperature of $10 \mathrm{~K}$ which is presented in Fig.7. In this case, the 13th torsional level was considered the ground one and in the formula (12) when calculating the partition function, the summation was performed from $i=13$ to $i=50$ with the step of 1. While comparing the spectra in Fig.5 and 7, it should be noted that the torsional spectrum of the cis-transformer is considerably richer. At least four absorption bands have significant intensity in the calculated torsional IR spectrum of the cis-transformer of MD. These bands have maxima near 282.9, 300.4, 377.4 and $397.6 \mathrm{~cm}^{-1}$ and are due to transitions $14 \Rightarrow 27,13 \Rightarrow 28,14 \Rightarrow 35$ и $13 \Rightarrow 38$ respectively.

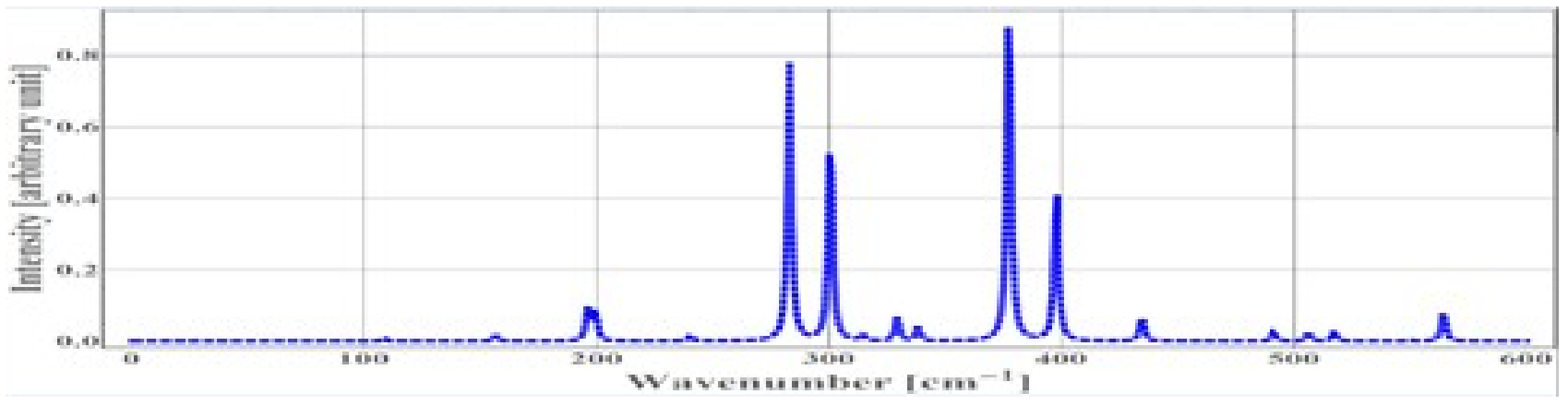

Figure 7. The torsional IR spectrum of the cis-transformer of the MD molecule at the temperature of $10 \mathrm{~K}$ calculated in the CBS approximation.

We also take into account that the 3rd torsional energy level is metastable since its multiplicity differs from the multiplicity of the second and ground torsional levels. This means that optical deactivation of an excited antisymmetric torsional vibration in the trans-conformer is forbidden by the selection rules inherent in the spin wave function. It is known, for example, that in the absence of external influences, water in the ortho-state can exist for a very long time $[49,50,51]$. With this in mind, the torsional spectrum of the trans conformer of MD was calculated for which the 3rd torsional level is ground at a temperature of $30 \mathrm{~K}$. In this case, in the formula (12) when calculating the partition function, the summation was performed from $i=13$ to $i=50$ with the step of 1 . It is presented in Fig. 8 . 


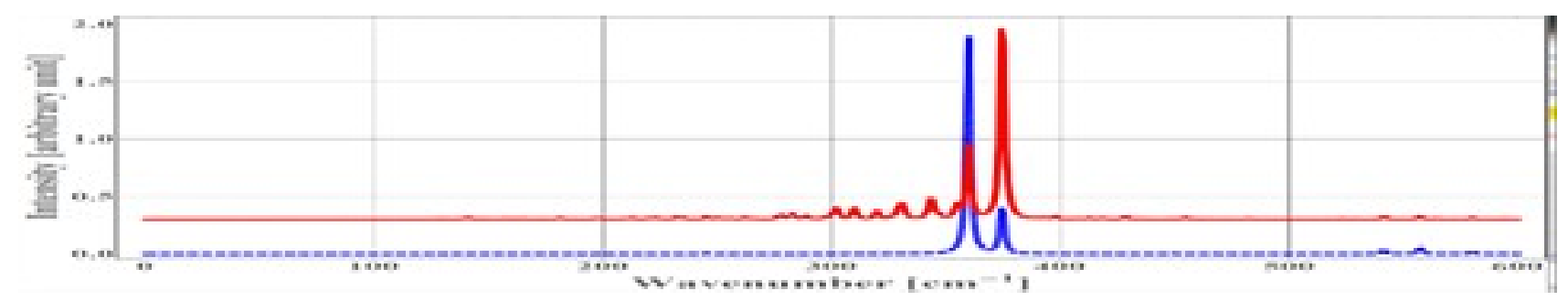

Figure 8. Torsional IR spectra of the trans-conformer of the MD molecule at the exited antisymmetric torsional state (third state) at the temperature of $30 \mathrm{~K}$ calculated in the approximation of the CBS limit.

As can be seen from Fig.8, in this case, the calculations predict the presence of two intense absorption bands with maxima near 359.7 and $374.5 \mathrm{~cm}^{-1}$ in the torsional spectrum, which are due to transitions $3 \Rightarrow 9,4 \Rightarrow 10$ and $5 \Rightarrow 11,6 \Rightarrow 12$ respectively. It should be noted that in the IR spectrum of the trans-conformer in the usual case when the first torsional state is ground at $30 \mathrm{~K}$ the second band is absent since the 5th and 6th torsional levels are located 391 $\mathrm{cm}^{-1}$ above the ground state and at $30 \mathrm{~K}$ these levels are not populated. In contrast, in the transconformer of MD, which is stuck in the third excited torsional state, levels 5 and 6 turn out to be occupied, since they are only $57 \mathrm{~cm}^{-1}$ higher than the third torsional state. However, the situation under consideration may be of greater interest in relation to the tunneling frequency between the 3rd and 4th torsional states. According to the calculations in the CBS limit, the value of the tunneling frequency, in this case, is equal to $1,102279 * 10^{-5} \mathrm{~cm}^{-1}$ or $0,33045 \mathrm{MHz}$.

It is clear that these three absorption bands $\left(397,8 \mathrm{~cm}^{-1} 359,7\right.$ и $\left.374,5 \mathrm{~cm}^{-1}\right)$ for the transconformer and four absorption bands (282.9, 300.4, 377.4 и $\left.397.6 \mathrm{~cm}^{-1}\right)$ for the cis-conformer can serve as reference points for searching MD in the Earth atmosphere and interstellar space. It is worth noting that calculated intensities of the tunnelling transitions in the ground and third torsional states of the trans-conformer are weak enough due to very little permanent dipole moment, while the respective tunnelling transition in the cis-conformer is forbidden due to the changes of the symmetry of the spin wave functions.

\section{CONCLUSIONS}

The conformation states of the MD molecule have been analysed with the use of MP2/ccpVQZ level of theory.

The torsional spectra of trans- and cis-conformers of MD have been calculated for the first time with the use of the several levels of theory. It has been shown that computed values of the energies of the stationary torsional states are sensitive to the used levels of theory and density of the grid, at the nodes of which the potential energy and the values of the kinematic coefficients are evaluated. At the same time, there is a clear agreement between the frequency 
values of the torsional vibrations in the MD molecule calculated at different levels of theory. However, the computed values of frequency of the torsional vibrations obtained in CBS limit should be considered as the most accurate ones.

The torsional and spin wave functions of the MD molecule were classified based on the irreducible representations of the $\mathrm{C}_{2 \mathrm{~V}}(\mathrm{M})$ molecular symmetry group.

The MD molecule shows complex torsional vibrational dynamics. Among fifty considered excited torsional states there are vibrations belonging to a) only trans-conformer, b) only cis-conformer and c) both conformers, as well. Based on the torsional quantum numbers represented in Table 3 one can recognize the fundamental, overtones and combination torsional vibrations of the two hydroxyl groups in the MD molecule.

The tunnelling frequencies for the ground and metastable states of the trans-conformer as well as for cis-conformer of the MD molecule were calculated. In the last case, the tunnelling is forbidden due to the different symmetry of the spin wave functions of the 13 and 14 torsional states. The tunnelling for the ground and metastable states of the trans- conformer of the MD molecule is not forbidden by any symmetry restrictions. Thus calculated tunnelling frequencies (0.0633 and $0,33045 \mathrm{MHz}$ ) in combination with calculated frequencies of the torsional vibrations will be useful for the searching of the MD molecule in the interstellar space as well as in the Earth atmosphere.

\section{References}

[1] L. Radom, W.A. Lathan, W.J. Hehre, J.A. Pople, Australian J. Chem. 25 (1972) 1601-1612.

[2] G.A. Jeffrey, J.A. Pople, L. Radom, Carbohydrate Res. 25 (1972) 117-131.

[3] I.H. Williams, D. Spangler, D.A. Femec, G.M. Maggiora, R.L. Schowen, J. Am. Chem. Soc. 105 (1983) 31-40.

[4] M. Rodler, Chem. Phys. 105 (1986) 345-353.

[5] M.R. Hazra, J.S. Francisco, A. Sinha, J. Phys. Chem. A, 117 (2013) 11704-11710.

[6] W.A. Schutte, L.J. Allamandola, S.A. Sandorf, Iracus 104 (1993) 118-137.

[7] D.R. Kent, S.L. Widicus, G.A. Blake, W.A. Goddard, J. Chem. Phys. 119 (2003) 5117-5120.

[8] C. Barrientos, P. Redondo, H. Martinez, A. Largo, Astrophys.J. 784:132 (2014).

[9] R.T. Garrod, S.L. Widius Weaver, E. Herbst, Proc. Int. Astrophys. Union 4 (2008) 123-124.

[10] F. Duvernay, A. Rimola, P. Theule, G. Danger, T. Sanchez, T. Chivassa, Phys. Chem. Chem. Phys 16 (2014) 24200-24208.

[11] C. Lugez, A. Schriver, R. Levany, L. Schriver-Mazzuoli, Chem. Phys. 181 (1994) 129-146. 
[12] R.S. Ryabova, G.I. Voloshenko, V.D. Maiorov, G.F. Osipova, Russ. J. Appl. Chem. 75 (2002) 22-24.

[13] H. Matsuura, M. Yamamoto, H. Murata, Spectrochim. Acta 36A (1980) 321-327.

[14] G.R. Möhlmann, J. Ram. Spectr. 18 (1987) 199-203.

[15] N. Lebrun, P. Dhamelincourt, C. Focsa, B. Chazallon, J.L. Destombes, D. Prevost, J. Ram. Spectr. 34 (2003) 459-464.

[16] B. Hanoune, L. Paccou, P. Delcroix, Y. Guinet, J. Ram. Spectr. 42 (2011) 1202-1204.

[17] P. Delcroix, M. Pagliai, G. Cardini, D. Bégué, B. Hanoune, J. Phys. Chem. A 119 (2015) 290-298.

[18] A. Vila, L. Estévez, R.A. Mosquera, J. Phys. Chem. A 115 (2011) 1964-1970.

[19] B. M. Hays, S.L. Widicus Weaver, J. Phys. Chem. A, 117 (2013) 7142-7148.

[20] G. Hetzer, P. Pulay, H.J. Werner, Chem. Phys. Lett. 290 (1998) 143-149.

[21] M. Schutz, G. Hetzer, H.J. Werner., J. Chem. Phys. 111 (1999) 5691-5705.

[22] D.E. Woon, T.H. Dunning Jr., J.Chem.Phys. 98 (1993) 1358-1371.

[23] http://www.msg.ameslab.gov/GAMESS/GAMESS.html

[24] E.B. Wilson, J.J.C. Decius, P.C. Cross, Molecular Vibration Dover Publications, Inc., New York, 1955.

[25] T.J. Lukka, J. Chem. Phys. 102 (1995) 3945-3955.

[26] J. Makarewicz, A. Skalozub, Chem. Phys. Lett. 306 (1999) 352-356.

[27] G.A. Pitsevich, A.E. Malevich, J. Appl. Spectr. 82 (2015) 540-553.

[28] R.A. Kendall, T.H. Dunning Jr., R.J. Harrison, J. Chem. Phys. 96 (1992) 6796-6806.

[29] A. Halkier, T. Helgaker, P. Jørgensen, W. Klopper, H. Koch, J. Olsen, A.K. Wilson, Chem. Phys. Lett. 286 (1998) 243-252.

[30] M. Okoshi, T. Atsumi, H. Nakai, J. Comp. Chem. 36 (2015) 1075-1082.

[31] D.O. Harris, G.G. Enderholm, W.D. Gwinn, J. Chem. Phys. 43 (1965) 1515-1517.

[32] A.S. Dickinson, P.R. Certain, J. Chem. Phys. 49 (1968) 4209-4211.

[33] R. Meyer, J. Chem. Phys. 52 (1970) 2053-2059.

[34] J.C. Light, I.P. Hamiltonian, J.V. Lill, J. Chem. Phys. 82 (1985) 1400-1409.

[35] D.T. Colbert, William H. Miller, J. Chem. Phys. 96 (1992) 1982-1991

[36] G.A. Pitsevich, A.E. Malevich, J. Appl. Spectr. 82 (2016) 893-900.

[37] Mathematica, Wolfram Research, Inc., http://www.wolfram.com/mathematica

[38] H.C. Longuet-Higgins, Mol. Phys. 6 (1962) 445-460.

[39] J.T. Hougen, J. Chem. Phys. 37 (1962) 1433-1441.

[40] P. R. Bunker and P. Jensen, Molecular Symmetry and Spectroscopy, NRC Research Press, Ottawa (1998). 
[41] J. Antony, G. von Helden, G. Meijer, B. Schmidt, J. Chem. Phys. 123 (2005) 0143051.

[42] J. O. Jung, R. B. Gerber, J. Chem. Phys. 105 (1996) 10332-10348

[43] G. A. Pitsevich, A. E. Malevich, I.Yu. Doroshenko, E. N. Kozlovskaya, V. E. Pogorelov, V. Sablinskas, V. Balevicius, Spectrochim. Acta, Part A 120 (2014) 585-594.

[44] G. A. Pitsevich, A. E. Malevich, E. N. Kozlovskaya, E. Mahnach, I.Yu. Doroshenko, V. E. Pogorelov, L.G.M. Pettersson, V. Sablinskas, V. Balevicius, J. Phys. Chem. A 121 (2017) 21512165 .

[45] Carlo Di Lauro, Rotational Structure in Molecular Infrared Spectra, Elsevier, 2013.

[46] G. Duxbury, Vibration-Rotation Spectroscopy, John Wiley \& Sons, LTD, 2000 p.25.

[47] J.M. Flaud, C. Camy-Peyret, J. Mol. Spectr. 55 (1975) 278-310.

[48] G. A. Pitsevich, I.Yu. Doroshenko, A. E. Malevich, E.Z. Shalamberidze, V.V. Sapeshko, V. E. Pogorelov, L.G.M. Pettersson, Spectrochim. Acta, Part A 172 (2017) 83-90.

[49] G. A. Pitsevich, E.Z. Shalamberidze, A. E. Malevich, V. Sablinskas, V. Balevicius, L.G.M. Pettersson, Mol. Phys. 115 (2017) 2605-2613.

[50] L. Abouaf-Marguin, A.M. Vasserot, C. Pardanaut, J. Chem. Phys. 130 (2009) $054503-054504$.

[51] L. Abouaf-Marguin, A.M. Vasserot, C. Pardanaut, X. Michaut, Chem. Phys. Lett. 480 (2009) 82-85. 